\title{
Detection of Overload Generated Faults in Electro-Hydrostatic Actuators
}

\author{
Lőrinc Márton and Andreas Varga \\ Institute of Robotics and Mechatronics \\ DLR - German Aerospace Center \\ Münchenerstr. 20, D-82234 Weßling \\ Phone: +49 (8153) 28-1361 Fax: +49 (8153) 28-1441 \\ E-Mails: marton.loerinc@dlr.de (martonl@ms.sapientia.ro) and andreas.varga@dlr.de
}

\begin{abstract}
This paper proposes a method for detecting faults generated by increased loads in a class of mechanical control systems. It is considered that the load (input disturbance) is unmeasurable, only its bounds corresponding to normal operation are known. To solve the fault detection problem, a detector was proposed for a class of linear control systems that can be implemented with low computational costs. The method is applicable for electro-hydrostatic actuators (EHA) with unknown load. The proposed fault detection approach is validated through simulations for jamming detection of aircraft control surfaces driven by EHA.
\end{abstract}

Index Terms-Fault detection, Hydraulic actuator, Disturbance estimation, Mechanical jamming

\section{INTRODUCTION}

The electro-hydrostatic actuators (EHA) represent a novel approach to steer the control surfaces of an aircraft. With this actuating method the hydraulic power supply and many hydraulic pipes can be omitted from the aircraft, because the actuator also generates the necessary hydraulic pressure for moving the piston (Power By Wire technology). The new generation of Airbus passenger aircrafts (A380) are also applying EHAs in the backup system of control surface actuation.

During their operation, the electrical and hydraulic actuators, which work in hazardous environments, are exposed to various types of faults. Fast and reliable detection of these faults is indispensable especially in safety critical systems such as aircrafts. The classical approach for detecting faults is to run a reliable process model parallel with the real process with same inputs. Based on the difference between the real output and the model output a residual signal is generated. If the residual is greater then a given threshold value, the system is in fault mode.

The model uncertainties and unmeasurable disturbances could severely influence the performances of fault detectors, leading to undetected faults or false alarms. It is why robust fault detection methods have to be developed [1]. The classical approach is to decouple the disturbances on the generated residuals by applying linear or nonlinear unknown input observers [2]. When the exact decoupling is not solvable, approximate decoupling methods can be applied, based on different optimization methods, see e.g. [3].
Another approach to deal with unmeasurable disturbances is to use extended state estimators that incorporate the disturbance into the estimator model, and tries to estimate it as an unknown state. In [4] it was shown, how a general structure observer can be extended for input disturbance estimation. The paper [5] proposes PI and PD type observers to detect constant input and sensor faults. In the study [6] the disturbance observer based fault detection was extended for a class of nonlinear systems.

The introduction of fault detection algorithms in aircraft control systems is indispensable. In the classical Fly By Wire systems the servo controlled hydraulic actuators are wide spread. These actuators have an external hydraulic power supply which generates the necessary pressure for driving the piston. The hydraulic flow in the cylinders is controlled by an electro-mechanical servo valve driven by a servo controlled motor [7], [8]. Several fault detection algorithms were developed for these type of hydraulic actuators. The most popular approaches are based on Extended Kalman Filters or on nonlinear observers [9]-[13]. These observers can be applied in state estimation or parameter estimation approach, depending on the formulated fault detection problem. However most of these methods assume that more than one internal states of the system (e.g. chamber pressure difference) can be measured and the effect of the external forces were considered known or can be neglected.

In this work we considered the case when on the rod position is the only measurable state of the actuator and the external load generated force (which is considered as an input disturbance) is unmeasurable, only its limits corresponding to normal operation are known. The proposed fault detection method is formulated for a general class of linear systems, to which the EHA belongs.

The remaining part of the paper is organized as follows: Section II introduces the proposed fault detection method for a general class of linear systems. Section III presents the dynamic model of the electro-hydrostatic actuators, based on which the fault detector can be designed. Section III presents, how the fault detection method can be applied for electro-hydrostatic actuators and it contains simulation results. Finally, Section V sums up the conclusions of this 
work.

\section{Disturbance Estimation Based Fault DETECTION}

Consider a stable linear control system in the form:

$$
\begin{aligned}
\dot{x} & =A x+B(u+d+f) \\
y & =C x,
\end{aligned}
$$

where $x \in R^{n}$ is the vector of states, $y \in R^{p}$ is the vector of outputs, $u, d, f \in R^{m}, m \leq p$ are the vectors of control inputs, disturbances and faults respectively. Assume that $(A, C)$ is observable and $(A, B)$ is controllable. The disturbance and fault vectors may be state dependent.

Since the fault $f$ and the disturbance $d$ enters in the same channel in the model, the effect of the disturbance on residual cannot be decoupled [1].

Rewrite the vector of faults and disturbances in the following form:

$$
d+f=: d_{0}+\delta d(t)
$$

where $d_{0}$ denotes the dominant low frequency component in the signal and $\delta d$ is a zero mean value high frequency disturbance.

Now assume that the absolute value of the $i$ th component of the input disturbance vector (e.g. external load on the mechanical system) during normal operation lies in an apriori known domain given by, i.e. $\left|d_{i}\right| \leq d_{M A X i}(y, u)$. Generally it can be assumed that the magnitude of the fault $f$ is greater than the magnitude of the disturbance signal $d$. The limit value $d_{M A X i}$ can be exceeded when a fault appears in the system, for example due to increased friction or mechanical jamming. Hence based on the estimated input disturbance, decision signals for overload $\left(r_{O L i}\right)$ can be defined as follows:

$$
r_{O L i}=\left\{\begin{array}{l}
1, \text { if }\left|\hat{d}_{0 i}\right|>d_{M A X i}(y, u) \\
0, \text { otherwise. }
\end{array}\right.
$$

Here $\hat{d}_{0 i}$ denote the estimated value of $d_{0 i}$ (the residual signal).

\section{A. PI Observer Based Design}

Assume that $d_{0}$ is constant. Rewrite the equation (1) as follows:

$$
\begin{aligned}
\left(\begin{array}{c}
\dot{x} \\
\dot{d}_{0}
\end{array}\right) & =\underbrace{\left[\begin{array}{cc}
A & B \\
0 & 0
\end{array}\right]}_{A_{\text {ext }}}\left(\begin{array}{c}
x \\
d_{0}
\end{array}\right)+\underbrace{\left[\begin{array}{c}
B \\
0
\end{array}\right]}_{B_{\text {ext }}} u+\left[\begin{array}{c}
B \\
0
\end{array}\right] \delta d, \\
y & =\underbrace{\left[\begin{array}{cc}
C & 0
\end{array}\right]}_{C_{\text {ext }}}\left(\begin{array}{c}
x \\
d_{0}
\end{array}\right) .
\end{aligned}
$$

Based on this extended model, a PI type observer can be developed in the form:

$$
\left(\begin{array}{c}
\dot{\hat{x}} \\
\dot{\hat{d}}_{0}
\end{array}\right)=\left[A_{\text {ext }}-K C_{\text {ext }}\right]\left(\begin{array}{c}
\hat{x} \\
\hat{d}_{0}
\end{array}\right)+B_{\text {ext }} u+K y,
$$

where $A_{\text {ext }}, B_{\text {ext }}$ and $C_{\text {ext }}$ denote the extended system matrices from the model (4).

$K$ is a gain matrix which stabilizes $\left[A_{\text {ext }}-K C_{\text {ext }}\right]$. $K$ has to be designed such that to deal with the high frequency component of the disturbance $(\delta d)$ as well. It can be exploited that the mean value of $\delta d$ is zero. When $\delta d$ is independent on the system states and it can be approximated with a multivariate normal distribution $w(t)$, i.e. $\delta d(t) \approx w(t)$, standard Kalman filter design procedure [14] can be applied to obtain $K$. Otherwise assume that the time dependent disturbance can be approximated as follows: $B \delta d \approx x \dot{\xi}(t)+\dot{w}(t)$, where $w(t) \in R$ and $\xi(t) \in R$ are generated by exogenous Wiener processes. In this case state estimator design procedures developed for Itô type processes can be applied, that minimize the effect of $w(t)$ and $\xi(t)$ on the estimation error $x-\hat{x}$, see for example [15], [16].

\section{B. Residual Generator Based Design}

In industrial practice simply implementable algorithms are required with low computational and implementation costs. For the implementation of the PI type observers the dimension of the dynamic system that generates the faults is $n+m$, where $n$ is the dimension of the state vector and $m$ is the dimension of the input disturbance vector.

In order to obtain a simplified model for estimating $d_{0}$, consider the model of the system (1) written in a transfer matrix form:

$$
y(s)=G(s) u(s)+G(s)\left(d_{0}(s)+\delta d(s)\right),
$$

where $G(s)=C(s I-A)^{-1} B$.

The estimation of $d_{0}$ can be formulated as a fault detection problem, i.e. find a residual signal $r(s)$ in function of $u(s)$ and $y(s)$ such that $r(s)=M(s) d_{0}(s)$, where $M(s)$ is a diagonal transfer matrix with stable, unit steady state gain transfer functions in the diagonal. Assume the residual generator in the form:

$$
r(s)=\left[Q_{y}(s) Q_{u}(s)\right]\left[\begin{array}{c}
y(s) \\
u(s)
\end{array}\right]
$$

where $Q_{y}(s)$ and $Q_{u}(s)$ are transfer matrices to be determined.

The residual generator can also be written as:

$$
\begin{aligned}
& r(s)=\left[Q_{y}(s) Q_{u}(s)\right]\left[\begin{array}{cc}
G(s) & G(s) \\
I & O
\end{array}\right]\left[\begin{array}{c}
u(s) \\
d_{0}(s)+\delta d(s)
\end{array}\right] \\
& \quad=\left[Q_{y}(s) G(s)+Q_{u}(s) Q_{y}(s) G(s)\right]\left[\begin{array}{c}
u(s) \\
d_{0}(s)+\delta d(s)
\end{array}\right] .
\end{aligned}
$$

The formulated fault detection problem is equivalent with:

$$
\left[Q_{y}(s) G(s)+Q_{u}(s) Q_{y}(s) G(s)\right]=[O M(s)] \text {, }
$$

from where the transfer matrices of the residual generator can be obtained:

$$
\left\{\begin{array}{l}
Q_{y}(s)=M(s) G^{\dagger}(s) \\
Q_{u}(s)=-M(s)
\end{array}\right.
$$


where $G^{\dagger}(s)$ is the left inverse of $G(s)$. Hence the residual generator has the form:

$$
r(s)=M(s) G^{\dagger}(s) y-M(s) u
$$

The stable, unit steady state gain transfer matrix $M(s)$ has to be chosen such that $M(s) G^{\dagger}(s)$ is proper and stable. It should also have a low-pass filter behavior to attenuate the effect of the high frequency noise $(\delta d)$ on the residual.

The residual generator above may have high degree in order to achieve the desired filtering properties and to ensure that $M(s) G^{\dagger}(s)$ is proper. In order to obtain a simply implementable form for the residual generator, model reduction techniques [17] can be applied for $G(s)$ and the residual generator can be designed based on a reduced model $G_{r}(s)$. In this case it should be checked that the following norms will not exceed some given reasonable limits.

$$
\begin{aligned}
& \sup _{0 \leq \omega<\omega_{f}}\left\|M(j \omega)-M_{r}(j \omega)\right\|_{2}, \\
& \sup _{0 \leq \omega<\omega_{f}}\left\|M(j \omega) G^{\dagger}(j \omega)-M_{r}(j \omega) G_{r}^{\dagger}(j \omega)\right\|_{2},
\end{aligned}
$$

where $M_{r}(s)$ is designed based on the model $G_{r}(s)$ such to have similar filtering properties as $M(s) . \omega_{f}$ denotes here the angular frequency over which the inputs are sufficiently attenuated due to the low pass filter property of the residual generator.

For example, assume that the control system under investigation has a fast dynamics, hence it can be approximated as $G_{r}(s)=G(0)$, and $G(0)$ is left invertible. With this assumption the transfer matrix $M_{r}(s)$ can be chosen as a diagonal matrix with first order filters in the diagonal in the form $M_{r}(s)=\operatorname{diag}\left[\frac{k_{f i}}{s+k_{f i}}\right]$, where $k_{f i}>0, i=\overline{1, m}$. With this approximation in time domain the residual generator has the following easily implementable form:

$$
\dot{r}=K_{f}\left(G(0)^{\dagger} y-(u+r)\right),
$$

where $K_{f}=\operatorname{diag}\left[k_{f i}\right]$. Note that with this filter the input $u$ will not be completely decoupled from the residual $r$. However if closed loop control system is assumed with piecewise-constant reference signal as input, the residual generator (11) can be applied with good decoupling performances.

\section{EHA Model for FAUlt Detection}

The electro-hydrostatic actuator has a sealed pressurized housing filled with hydraulic fluid. A bi-directional motor is immersed in the fluid and drives a pump for exchanging fluid via a hydraulic circuit between the chambers of the actuator. The chambers are separated by a piston and consider that the rod of the piston actuates an aircraft control surface.

During aircraft operation the aerodynamic effects generate high magnitude forces which act as an external load force on the actuator. These forces depend on the actuator position, direction of the actuator's motion, and external factors such as aircraft altitude and speed.

\section{A. Basic Equations}

The dynamic model of the actuator can be derived based on the Newtonian motion of the piston's rod, on the dynamics of the hydraulic fluid pressure difference in the actuator chambers and on the dynamical equation of the electrical motor that actuates the pump. Consider that the EHA actuates a control surface of an aircraft (see Figure 1).

The motion of the rod is described by the following equation:

$$
M \ddot{x}+F_{f}(\dot{x})+F_{\text {aero }}=S \Delta P,
$$

where $x$ denote the rod position, $\Delta P$ is the pressure difference between the two chambers of the actuator, $F_{\text {aero }}$ is the aerodynamic force applying on the control surface, $F_{f}$ is the friction induced damping. $S$ is the area of the piston surface, $M$ is the mass of the actuator's rod and its load.

The pressure differential dynamics is given by (see e.g. [18], [19]):

$$
C_{H}(x) \Delta \dot{P}=Q_{f}-S \dot{x},
$$

where $Q_{f}$ denotes the flow in the hydraulic circuit and $C_{H}(x)$ is the hydraulic capacity that can be calculated as: $\frac{1}{C_{H}(x)}=\frac{1}{C_{1}(x)}+\frac{1}{C_{2}(x)} ; C_{1}(x)=\frac{V_{01}+S x}{B} ; C_{1}(x)=$ $\frac{V_{02}+S(L-x)}{B}$. The parameters in the relations above are: $V_{01}, V_{02}$ initial chamber volumes, $B$ bulk modulus of the hydraulic fluid, $L$ internal length of the actuator cylinder.

The flow $\left(Q_{f}\right)$ through the hydraulic circuit can be assumed to be proportional with the angular speed $(\omega)$ of the electrical motor which generates the pressure difference [20], [21]. In order to sustain the closed loop hydraulic circuit of EHA, a refeeding circuit with a pressurized accumulator and check valves is necessary. The flow from/to the accumulator can be assumed proportional with the pressure difference $\Delta P[18],[22]$.

$$
Q_{f}=D \omega-K_{L} \Delta P,
$$

where $D$ is the displacement of the pump divided by $2 \pi$, $K_{L}$ is the leakage coefficient.

The model for the electrical motor that actuates the pump (Direct Current speed controllable motor is assumed with neglected electrical time constant, $L_{m} / R<<$ ) reads as:

$$
\begin{aligned}
& J \dot{\omega}=K_{i} i-\tau_{L}, \\
& i=\frac{1}{R}\left(u_{M}-K_{e m f} \omega\right), \\
& \tau_{L}=K_{D P} S \Delta P,
\end{aligned}
$$

where $\omega$ is the angular speed of the electrical motor, $i$ is the current through the motor, $u_{M}$ is the input voltage of the motor, $\tau_{L}$ is the motor's load generated torque (proportional with the generated pressure difference). Parameters: $J$ is the inertia of the rotor and the motor load, $K_{i}$ is the torque constant, $K_{e m f}$ is the speed constant, $R$ is the motor resistance, $L_{m}$ is the motor inductance, $K_{D P}$ is the pressure difference generated load constant. 


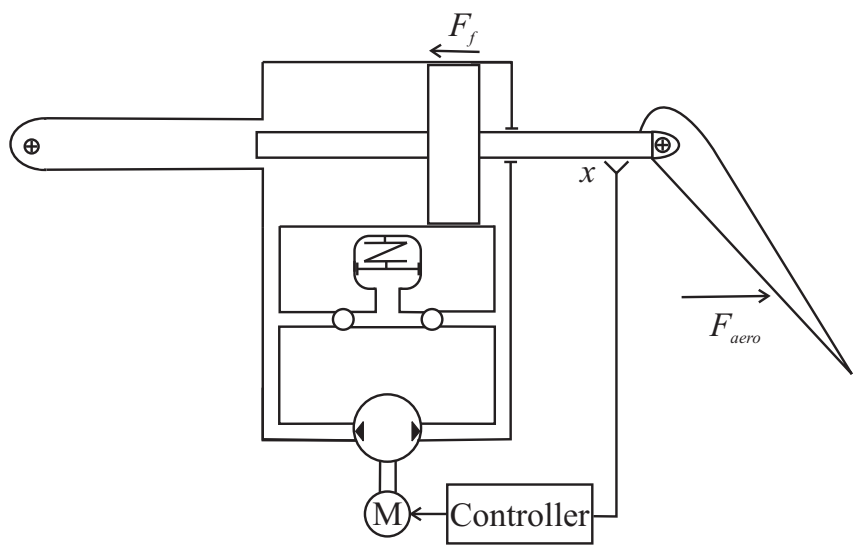

Fig. 1. Schematic of control surface actuation with EHA

The model of the actuator can be summarized as follows:

$$
\left\{\begin{array}{l}
M \ddot{x}+F_{f}(\dot{x})+F_{\text {aero }}=S \Delta P \\
C_{H}(x) \Delta \dot{P}=D \omega-S \dot{x}-K_{L} \Delta P \\
J \dot{\omega}=K_{i} \frac{1}{R}\left(u_{M}-K_{e m f} \omega\right)-S K_{D P} \Delta P .
\end{array}\right.
$$

Note that during modeling some factors, such as friction induced nonlinearities, the internal leakage between the actuator chambers were neglected. In the case of more elaborate nonlinear modeling these factors should also be taken into consideration [23]. In this work it was considered that the amount of force generated by the aerodynamic forces has much greater influence on the dynamics than the neglected factors.

\section{B. Simplified model for Fault Detection}

Consider that the friction in the actuator can be approximated with a viscous friction model $\left(F_{f}(\dot{x})=F_{V} \dot{x}, F_{V}>\right.$ 0 ) and the time constant of the motor and the hydraulic time constant can be neglected related to the main mechanical time constant of the system. Hence by assuming that $\Delta \dot{P}=0$ and $\dot{\omega}=0$ from (16) results:

$$
S \Delta P=\frac{K_{i}}{R K_{S \Delta P}}\left(u_{M}-K_{e m f} \frac{S}{D} \dot{x}\right)
$$

where $K_{S \Delta P}=K_{\Delta P}+\frac{K_{I} K_{e m f} K_{L}}{R S D}$.

In aircraft fault detection systems the controller can also be introduced into the model that is used for fault detection [24], [25]. Assume that the position control of the actuator is solved by using a PD type control law: $u_{M}=K_{P}\left(x_{r e f}-\right.$ $x)-K_{D} \dot{x}, K_{P}, K_{D}>0$. Hence the simplified model of the actuator control system reads as:

$$
\begin{array}{r}
M \ddot{x}+\left(F_{V}+\left(K_{D}+K_{e m f} \frac{S}{D}\right) \frac{K_{i}}{R K_{S \Delta P}}\right) \dot{x}+ \\
\frac{K_{P} K_{i}}{R K_{S \Delta P}} x=\frac{K_{P} K_{i}}{R K_{S \Delta P}} x_{r e f}-F_{\text {aero }}+f,
\end{array}
$$

where $f$ is the force that generates the overload fault. During normal operation $f=0$.

\section{Input Disturbance and Jamming Fault}

The aerodynamic force, that acts on the actuator dynamics as an external load force, beside the actuator position depends on many actuator independent factors and it can hardly be measured during aircraft operation. It also depends on the sign of actuator velocity: when the control surface approaches zero position the aerodynamic force acts as a helping force; when the surface departs from zero position the force acts as a breaking force. Generally $F_{\text {aero }}$ can be considered as an unmeasurable input disturbance that depends on the states of the actuator and on external parameters as well.

In this work $F_{\text {aero }}$ in the following simplified form was assumed for simulation purposes:

$$
F_{\text {aero }}(x)=K_{\text {aero }}(p, x, \dot{x})|x|^{\alpha(p, \dot{x})} \operatorname{sgn}(x) \operatorname{sgn}(\dot{x})+F_{0}(p) \text {. }
$$

Here $F_{0}$ is the value of $F_{\text {aero }}$ in the zero position (when the control surface is in line with the wing), the vector $p$ incorporates mainly unmeasurable and actuator independent parameters and variables such as the aircraft altitude and speed, wind parameters, angle of attack of the airplane. $K_{\text {aero }}$ is a state dependent, time varying nonlinear gain. $\alpha$ is a parameter dependent exponent. It is considered that $\operatorname{sgn}(0)=0$.

In the case of jamming (overload type fault), the control surface stuck in a fixed position. A jammed control surface can severely affect the aircraft controllability and accordingly the safety of the flight operation. The root cause of this fault is generally the jamming or torsion of the actuator's rod or mechanical breakage of the actuated control surface. Based on the model (18) the jamming type fault in EHA can be modeled as:

$$
f=F_{\text {aero }}\left(x_{j a m}\right)+\frac{K_{P} K_{i}}{R K_{S \Delta P}}\left(x_{j a m}-x_{r e f}\right),
$$

where $x_{\text {jam }}$ is a constant jamming position.

A simple solution to detect jamming in control systems is based on the steady state error. If the difference between the real position and the prescribed position is greater than a given threshold after the settling time, it can be assumed that the system is in fault mode. However, in safety critical systems much faster detection time is required than the settling time, hence model based approaches have to be applied that can also deal with unmeasurable disturbances.

\section{Simulation Results}

The applicability of the fault detection method was tested on an EHA with similar parameters as it was given in [23]: $L=2.3 E-3 H, R=1.5 \Omega, K_{i}=0.2 \mathrm{Nm} / \mathrm{A}, K_{\text {emf }}=$ $4.2 E-4 \mathrm{Vs} / \mathrm{rad}, D=1.2 E-6 /(2 \pi) \mathrm{m}^{3} / \mathrm{rad}, M=$ $100 \mathrm{~kg}, S=71 \mathrm{E}-4 \mathrm{~m}^{2}, F_{V}=150 \mathrm{Ns} / \mathrm{m}, K_{\Delta P}=$ $.01 \mathrm{~m}$. For the control of the EHA a PD type control law was applied with $K_{P}=20000$ and $K_{D}=2000 \mathrm{~s}$.

The aerodynamic force (input disturbance) was generated by relation given in (19) with constant parameters as $K_{\text {aero }}=4 \mathrm{~N} / \mathrm{m}, F_{0}=25 \mathrm{~N}$ and $\alpha=1$. By working 


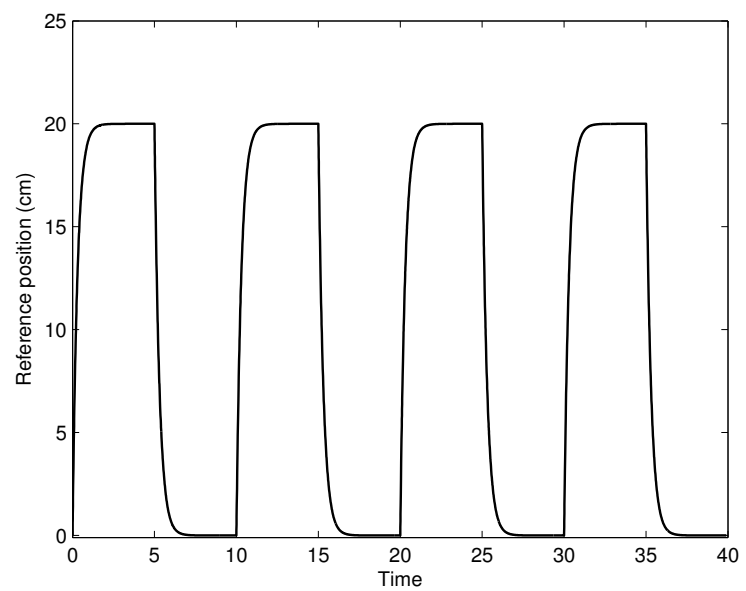

Fig. 2. Prescribed Position

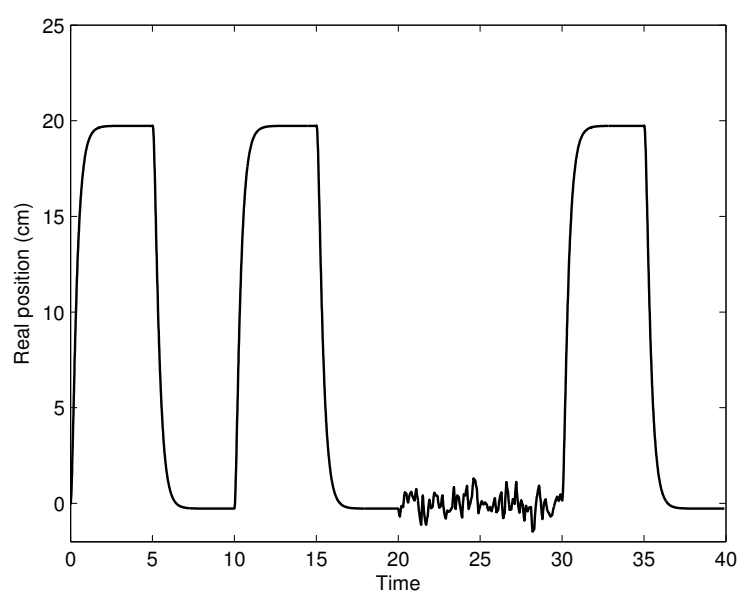

Fig. 3. Realized position

with constant parameters the accuracy of the aerodynamic model is compromised but, if it is assumed that the parameter vector $p$ is slowly varying, it is an acceptable simplification for demonstrating the performances of the fault detection method. The boundaries of the input disturbance were assumed as $-d_{M A X}<F_{\text {aero }}<d_{M A X}$, where $d_{M A X}=K_{M A X}|x|+F_{0 M A X}$ with $K_{M A X}=5 \mathrm{~N} / \mathrm{m}$, $F_{0 M A X}=100 \mathrm{~N}$.

The fault was injected into the control system as a mechanical jamming at zero position, and it was simulated using the relation (20). Over the constant jamming position a white noise type disturbance with maximum $1.5 \mathrm{~cm}$ amplitude was also added.

The fault detector (11) was constructed based on the model (18). The steady state gain (from $x_{r e f}$ to $x$ ) of the system is 1 and the load to be estimated is: $\frac{R K_{S} \Delta P}{K_{P} K_{i}}\left(F_{\text {aero }}+f\right)$. The amplification gain was chosen $K_{f}=20$ with which the cutoff frequency of the detector is around $20 \mathrm{~Hz}$.

Simulation results are shown in Figures 2 - 6. In all graphics the time unit is second (in horizontal axis). The prescribed position trajectory is a square signal with $20 \mathrm{~cm}$ amplitude and $10 s$ period, prefiltered using a first order filter with unit amplification and $0.3 s$ time constant. The simulated aerodynamic force (input disturbance) is shown in Figure 4. Since its value also depends on the sign of velocity, when the position approaches to zero, its value changes its sign.

In the Figure 3 it can be seen that the fault (jamming at 0 position) was injected into the system from $t=20 \mathrm{~s}$ to $t=30 \mathrm{~s}$. The Figure 5 shows the estimate of the input disturbance. When there is no fault, the proposed detection algorithm tracks the aerodynamic force with a good precision and in steady state the estimation error converges to zero. When the fault occurs, the estimated disturbance overpasses the given bound for the load, and the decision signal (see Figure 6) is active within $150 \mathrm{~ms}$ after the occurrence of the fault. (In Figure 5 the estimated disturbance has been saturated at $500 \mathrm{~N}$ ). In the time instant $t=25 \mathrm{~s}$ the reference position is set to zero again. Since the prescribed position is equal with the jamming position at $t=27.15 \mathrm{~s}$, the decision signal returns to zero.

\section{Conclusion}

A fault detection method was introduced for such control systems in which the faults and disturbances enter in the same input channel into the system. The residual is generated based on the estimated disturbance value by assuming that the bounds of the input disturbance are known. The fault detector is a first order stable system which can be implemented with low computational costs, hence it is applicable in industrial practice.

The proposed fault detection method was applied for mechanical jamming detection in aircraft control surfaces actuated by EHA. The input disturbance in this case is the aerodynamic force. Firstly a simplified model of the actuator was derived. Based on the model, a fault detector for jamming detection was designed and implemented. Simulation results show that the fault detector can precisely estimate the input disturbance and recognizes rapidly the fault state.

\section{ACKNOWLEDGMENT}

The research work of L. Márton was supported by Alexander von Humboldt Stiftung/Foundation scholarship for postdoctoral researchers.

\section{REFERENCES}

[1] J. Chen and R. J. Patton, Robust Model-Based Fault Diagonisis for Dynamic Systems. Boston: Kluwer Academic Publishers, 1999.

[2] P. M. Frank and X. Ding, "Survey of robust residual generation and evaluation methods in observer-based fault detection methods," Journal of Process Control, vol. 7, no. 6, pp. 403-424, 1997.

[3] A. Varga, "New computational approach for the design of fault detection and isolation filters," in Advances in Automatic Control The Kluwer International Series in Engineering and Computer Sciences, Kluwer Academic Publishers, 2003, pp. 367-381.

[4] C.-H. Lee, M.-S. Shin, H.-S. Kim, and S.-B. Kim, "Input disturbance estimation using a general structured observer," KSME International Journal, vol. 15, no. 2, pp. 1609-1615, 2001.

[5] Z. Gao and H. Wang, "Descriptor observer approaches for multivariable systems with measurement noises and application in fault detection and diagnosis," Systems \& Control Letters, vol. 55, pp. 304 $-313,2006$. 


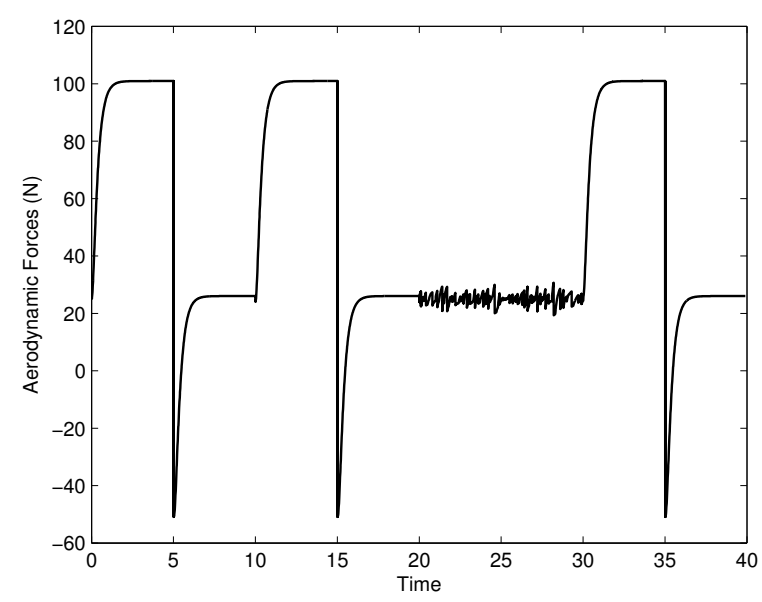

Fig. 4. Aerodynamic force

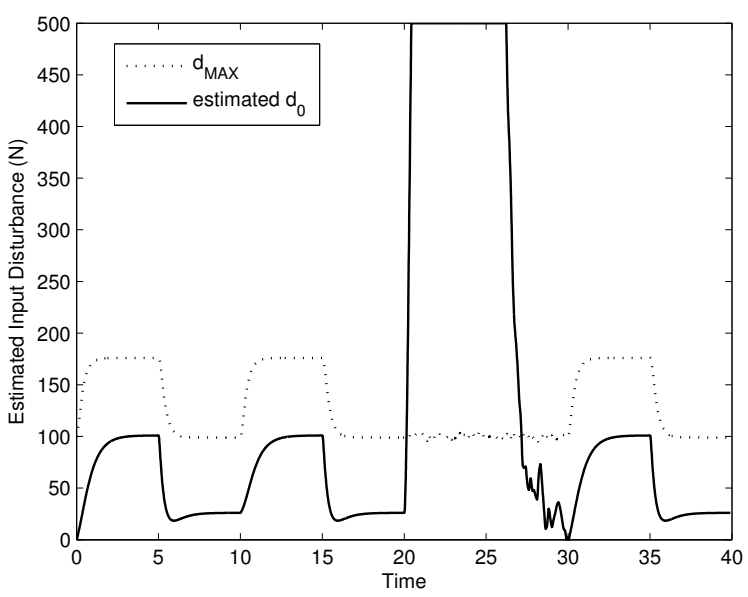

Fig. 5. Estimated Input Disturbance

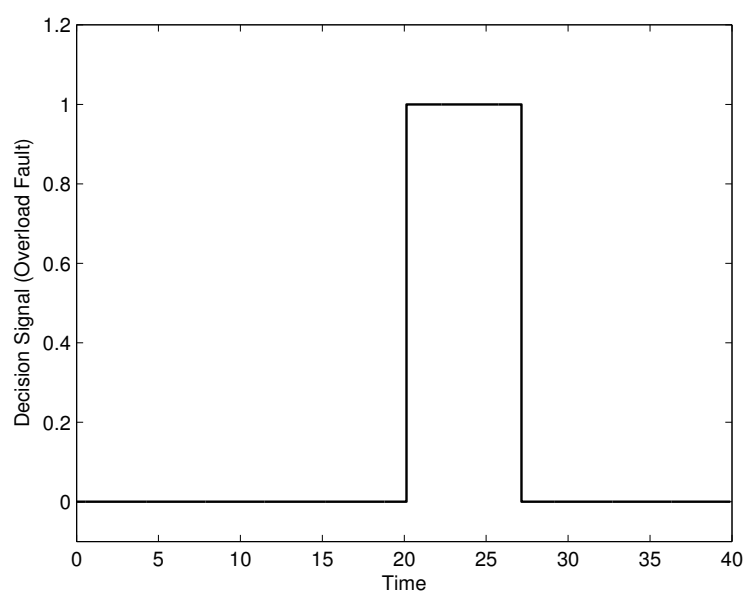

Fig. 6. Generated Decision Signal
[6] S. Cao and L. Guo, "Fault diagnosis with disturbance rejection performance based on disturbance observer," in Joint 48th IEEE Conference on Decision and Control and 28th Chinese Control Conference, Shanghai, P.R. China, December 2009.

[7] A. G. Loukianov, J. Rivera, Y. V. Orlov, and E. Y. M. Teraoka, "Robust trajectory tracking for an electrohydraulic actuator," IEEE Transactions on Industrial Electronics, vol. 56, no. 9, pp. 3523-3531, September 2009.

[8] L. Márton, S. Fodor, and N. Sepehri, "A practical method for friction identification in hydraulic actuators," Mechatronics, vol. 21, no. 1, pp. 350-356, February 2011.

[9] P. Garimella and B. Yao, "Fault detection of an electrohydraulic cylinder using adaptive robust observers," in Proceedings of IMECE04, 2004 ASME International Mechanical Engineering Congress and Exposition, Anaheim, California, USA, November 2004, pp. 1-10.

[10] X. Wang and V. L. Syrmos, "Fault detection, identification and estimation in the electro-hydraulic actuator system using ekf-based multiple-model estimation," in 16th Mediterranean Conference on Control and Automation, Ajaccio, France, June 2008, pp. 1693-1698.

[11] L. An and N. Sepehri, "Hydraulic actuator leakage quantification scheme using extended kalman filter and sequential test method," in Proceedings of the 2006 American Control Conference, Minneapolis, Minnesota, USA, June 2006, pp. 4424-4429.

[12] S. Gayaka and B. Yao, "Fault detection, identification and accommodation for an electro-hydraulic system: An adaptive robust approach," in Proceedings of the 17th World Congress The International Federation of Automatic Control, Soul, Korea, July 2008, pp. 13815-13 820.

[13] M. Leuschen, I. Walker, and J. Cavallaro, Nonlinear Fault Detection for Hydraulic Systems. Springer, 2003, pp. 169-191.

[14] C. Chui and G. Chen, Kalman Filtering. Berlin, Germany: Springer, 1999.

[15] V. Dragan and A.-M. Stoica, "Kalman-type estimation for systems corrupted with additive and multiplicative white noise," in Proc. of The International Symposium on System Theory, Automation, Robotics, Computers, Informatics, Electronics and Instrumentation, Craiova, Romania, October 2007.

[16] E. Gershon, U. Shacked, and I. Yaesh, $H_{\infty}$ Control and Estimation of State-multiplicative Linear systems. London, UK: Springer, 2005.

[17] G. Obinata and B. Anderson, Model Reduction for Control System Design. Springer, 2001.

[18] S. Frishemeier, "Electrohydrostatic actuators for aircraft primary flight control - types, modelling and evaluation," in 5th Scandinavian International Conference on Fluid Power, Linkping, Sweden, May 1997.

[19] H. Merritt, Hydraulic control systems. New York: John Wiley \& Sons, 1967.

[20] S. Habibi and A. Goldenberg, "Design of a new high-performance electrohydraulic actuator," IEEE/ASME Transactions on Mechatronics, vol. 5, no. 2, pp. 158-164, June 2000.

[21] V. Pastrakuljic, "Design and modeling of a new electro hydraulic actuator," Ph.D. dissertation, University of Toronto, 1995.

[22] R. Kang, Z. Jiao, S. Wu, Y. Shang, and J.-C. Mare, "The nonlinear accuracy model of electro-hydrostatic actuator," in IEEE Conference on Robotics, Automation and Mechatronics, Chengdu, China, November 2008, pp. 107-111.

[23] K. Rongjie, J. Zongxia, W. Shaoping, and C. Lisha, "Design and simulation of electro-hydrostatic actuator with a built-in power regulator," Chinese Journal of Aeronautics, vol. 22, pp. 700-706, 2009.

[24] P. Goupil, "Oscillatory failure case detection in the A380 electrical flight control system by analytical redundancy," in Seventeenth IFAC Symposium on Automatic Control in Aerospace, ICT, Universitas Studii Tolosana, France, June 2007.

[25] L. Lavigne, A. Zolghadri, P. Goupil, and P. Simon, "Oscillatory failure case detection for new generation Airbus aircraft: a modelbased challenge," in 47th IEEE Conference on Decision and Control, Cancun, Mexico, December 2008, pp. 1249-1254. 